\title{
Isolated Extensor Hallucis Longus Paralysis after Knee Arthroscopy: A case report
}

\author{
by Emmanuel P. Estrella, MD $^{1} \rrbracket$, Edgar Micheal T. Eufemio, MD $^{2}$
}

The Foot \& Ankle Journal 1 (10): 2

Arthroscopic surgery has gained widespread acceptance in the diagnosis and treatment of sportsrelated injuries to the knee. Neurovascular complications after knee arthroscopy are rare. The most common neural complications include injuries to the saphenous nerve and common peroneal nerve. Isolated paralysis of the extensor hallucis longus muscle has never been reported as a complication of arthroscopic knee surgery. However, it is not an uncommon complication in procedures around the proximal tibia or harvest of the fibula for bone grafting. We report a case of an isolated paralysis of the extensor hallucis longus muscle in a patient who had arthroscopic anterior cruciate ligament reconstruction and partial meniscectomy. A simple tendon transfer of the extensor hallucis longus to the extensor digitorum longus tendon allowed the hallux to dorsiflex and extend.

Key words: Nerve injury, arthroscopy, meniscectomy, tendon transfer, extensor hallucis palsy

Accepted: September, 2008

Published: October, 2008

This is an Open Access article distributed under the terms of the Creative Commons Attribution License. It permits unrestricted use, distribution, and reproduction in any medium, provided the original work is properly cited. @The Foot \& Ankle Journal (www.faoj.org)

\begin{abstract}
Arthroscopic knee surgery has been accepted as the standard treatment for anterior cruciate ligament (ACL) reconstruction and meniscus surgery. Although regarded as a minimally invasive surgery with low morbidity, complications still exist. ${ }^{1}$ Neural complications of knee arthroscopy range from $0.6 \%-2.5 \%$ in reported series. ${ }^{1,2,3}$ The nerve most commonly injured is the infrapatellar branch of the saphenous nerve on the medial aspect of the knee. This nerve can be injured with an incision during portal placement or harvest of the hamstring tendon for ACL reconstruction. ${ }^{4,5,6}$
\end{abstract}

\footnotetext{
Address correspondence to: Emmanuel P. Estrella, MD

Department of Orthopedics. Philippine General Hospital, Manila, Philippines 1000. Email: estee96@yahoo.com

Tel \#: (632) 5242203

${ }^{1}$ Staff surgeon, University of the Philippines-College of Medicine,

Department of Orthopedics, Philippine General Hospital, Manila, Philippines, 1000.

${ }^{2}$ Resident, University of the Philippines-College of Medicine,

Department of Orthopedics, Philippine General Hospital, Manila, Philippines, 1000.
}

ISSN 1941-6806

doi: $10.3827 /$ faoj.2008.0110.0002
Motor deficit from injury to the peroneal nerve after knee arthroscopy has been reported in a few case reports. ${ }^{4,7,8,9.10}$

Paralysis of the extensor hallucis longus (EHL) muscle has been frequently reported as a complication of procedures on the proximal part of the tibia, fibula or fibular graft harvest. ${ }^{11,12}$ It can be an isolated injury or part of a peroneal nerve injury. In our literature search, we found no reported case of isolated EHL paralysis after knee arthroscopy. The purpose of this report is to present an unusual injury from a common arthroscopic knee procedure. 


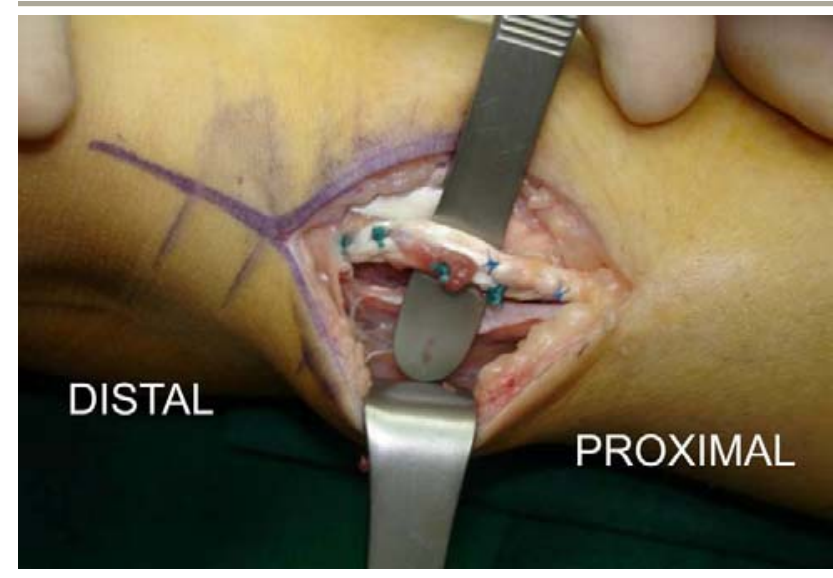

Figure 1 Intraoperative view of the transferred distal tendon of the EHL (extensor hallucis longus) to the common tendons of the EDL (extensor digitorum longus) via tendon weave.

\section{Case Report}

A 14 year-old male sustained a twisting injury of the left knee while playing basketball. A consultation by an orthopedic surgeon was performed a few weeks after the incident because of persistent left knee pain. Physical examination of the left knee showed a positive anterior drawer's and pivot shift test. A diagnosis of an ACL (anterior cruciate ligament) deficient knee was made and the patient was advised to undergo surgery. He had ACL reconstructive surgery using a hamstring graft and a partial lateral meniscectomy of the left knee. The patient noted loss of left big toe extension a day after the surgery. The lesser toe extensors and ankle dorsiflexion were intact. The patient was followed for several months and had no motor recovery.

The patient first consulted our clinic nine months after the arthroscopic ACL reconstruction and meniscectomy. He complained of the inability to extend the left big toe. Physical examination showed an MRC (Medical Research Council) muscle grade of $0 / 5$ for big toe extension or dorsiflexion. The lesser toes and ankle were graded $5 / 5$ on extension with no sensory deficit. There were no other motor and sensory abnormalities.

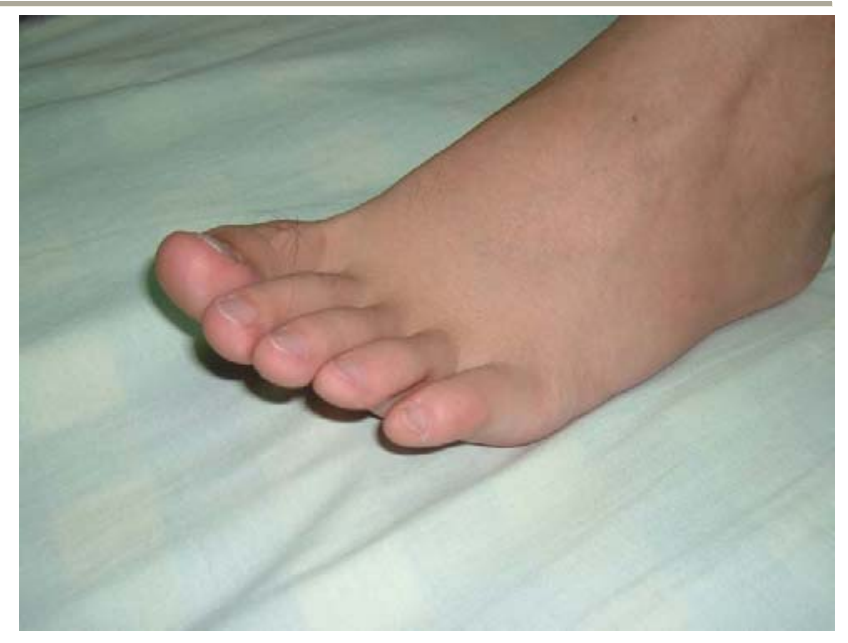

Figure 2 Motor function at one year post-op. Note the simultaneous extension of all five toes.

An electromyography-nerve conduction velocity (EMG-NCV) was done and showed complete denervation of the extensor hallucis longus muscle.

At one year after the ACL reconstruction, there was still no recovery of the dropped big toe and a surgical option was offered. Two years after the initial surgery, a decision to undergo surgery was made by the patient.

A tendon transfer was planned to restore the big toe extension. The tendons of the EHL and the EDL muscles were approached anteriorly, proximal to the extensor retinaculum. The EHL tendon was identified, transected and transferred to the common extensor digitorum longus tendons by a tendon weave suture technique under optimal tension with the ankle in plantigrade position. (Fig. 1) He was maintained in a cast boot for six weeks. He then started strengthening exercises for the transferred tendon-muscle unit with emphasis on simultaneous extension of all five toes. At the latest follow-up, at one year after the tendon transfer, simultaneous extension of the big and lesser toes was achieved with an MRC muscle grade of $4 / 5$ for big toe extension. (Fig. 2) 


\section{Discussion}

Isolated paralysis of the EHL muscle or as part of common peroneal nerve paralysis has been reported in procedures in the proximal part of the tibia such as high tibial or fibular osteotomies, tibial nailing or fibular graft harvest. ${ }^{11,12,13}$ Most of these paralyses were transient and resolve within a few months.

In arthroscopic knee surgeries, perhaps one of the most serious nerve complications is peroneal nerve paralysis. Injury to the peroneal nerve may be due to entrapment or nerve capture during lateral meniscus repair ${ }^{18,14}$, posterior trocar placement ${ }^{4}$, posterior capsular penetration ${ }^{9,10}$ or closed traction during intra-operative manipulation of the knee. $^{7}$ In cases of nerve entrapment by suture, release of the suture during re-exploration resulted in return of peroneal nerve function within six months. ${ }^{8,14}$ In one study ${ }^{4}$, there was a neuroma in continuity on reexploration that was resected and a nerve graft was performed. The authors suggested that the posterior placement of the trocar was the most probable cause of the neuroma formation. Other case reports showed peroneal nerve injury after arthroscopic meniscectomy alone. ${ }^{9,10}$ The authors attributed the injury from posterolateral capsular instrument penetration during meniscectomy as evidenced by a scarred posterolateral knee capsule and an unusually high bifurcation above the knee of the common peroneal nerve on reexploration. ${ }^{10}$

Deutsch, et al., ${ }^{15}$ investigated the anatomic variability of the peroneal nerve bifurcation as a possible risk factor for nerve injury in knee arthroscopy. They studied the anatomy of the common peroneal nerve bifurcation and noted that in $10 \%$ of the 70 cadaveric knees studied; bifurcation was noted to be proximal to the knee joint with an average distance of $7.5 \mathrm{~mm}$. Most bifurcation occurred at or below the fibular neck in $84.6 \%$ of cases. In the remaining $8.6 \%$, bifurcation occurred distal to the knee joint but proximal to the fibular neck.
A literature search revealed no isolated injury of the nerve to the EHL muscle after knee arthroscopy. The case presented here therefore is an example of an unusual injury from a common procedure.

The nerve to the EHL muscle as studied by Elgafy, et al., ${ }^{16}$ showed that the mean distance of the nerve from the deep peroneal nerve was $5.9 \pm$ $1.7 \mathrm{~cm}$ distally from the most palpable point of the fibular head, with an average length of $5.0 \pm$ $1.8 \mathrm{~cm}$. However, they also reported that among the thirty-three branches of the EHL that was dissected in thirty legs, twenty-seven (90\%) muscles had only one innervating branch.

A full explanation to the cause of the isolated paralysis of the EHL muscle could not be established. There were no surgical scars on the proximal lateral aspect of the leg that might be a cause of the injury to the nerve of the EHL. The injury might have occurred at the level of the knee joint where a capsular instrument penetration during meniscectomy was the cause, as reported by some authors ${ }^{9,10}$ in addition to a possible high bifurcation of the common peroneal nerve. The peroneal nerve is posterior and deep to the biceps femoris at the level of the joint line, and the inferior lateral geniculate vessels lie along the posterolateral aspect of the capsule at the meniscal attachment. Injury to the peroneal nerve was at most partial, and it is possible that the fascicle destined to innervate the EHL muscle was the one that was injured.

Some authors emphasized that a thorough understanding of the anatomy and anatomic variability of the neurovascular structures about the knee is essential in order to avoid neurovascular complications during knee arthroscopy., ${ }^{4,10,13}$ Flexion of the knee in $90^{\circ}$ or the so-called "figure-of-four" position should be done when arthroscopic instruments approach the posterolateral corner of the knee to decrease the risk nerve injury in cases of capsular penetration since the neurovascular structures are more distant from the knee capsule in this position. , $^{2,16}$ 
Likewise, caution must be exercised when motorized instruments or arthroscopic forceps are used in the posterolateral aspect of the knee.

In summary, this case highlights that even in common procedures such as arthroscopic ACL reconstruction and meniscectomy, complications do occur. The most devastating complications are functional loss to the foot and ankle because of peroneal nerve injuries. General principles in knee arthroscopy should be observed in order to avoid such complications.

\section{References}

1. Small NC. Complications in arthroscopic surgery performed by experienced arthroscopists. Arthroscopy 4 (3): 215-221, 1988.

2. Sanders B, Rolf R, McClland W et al: Prevalence of Saphenous Nerve Injury after Autogenous Hamstring Harvest: An Anatomic and Clinical Study of Sartorial Branch Injury. Arthroscopy 23 (9): 956-963, 2007.

3. Bernard M, Grosthuses-Spork M, Georgoulis A et al: Neural and Vascular complications of arthroscopic meniscal surgery. Knee Surg Sports Traumatol Arthrosc 2 (1): 14-18, March, 1994.

4. Krivić A, Staneg S, Zig R et al: Lesion of the Common Peroneal Nerve During Arthroscopy. Arthroscopy 19 (9): 1015-1018, 2003.

5. Luo H, Yu JK, Ao YF et al: Relationship between different skin incisions and the injury of the infrapatellar branch of the saphenous nerve during anterior cruciate ligament reconstruction. Chin Med J (Eng) 120 (13): 11271130, July, 2007.

6. Sherman OH, Fox JM, Snyder J et al: Arthroscopy: No problem surgery. An analysis of complications in two thousand six hundred and forty cases. JBJS 68A (2): 256265, 1986.

7. Johnson DS, Sharma DP, Bangash IH et al: Common Peroneal Nerve Palsy following Knee Arthroscopy. Arthroscopy 15 (7): 773-774, October, 1999.

8. Jurist K, Greene PW $3^{\text {rd }}$, Shirkhoda A: Peroneal nerve dysfunction as a complication of lateral meniscus repair: A case report and anatomic dissection. Arthroscopy 5 (2): 141 147, 1989.

9. Rodeo SA, Sobel, Weiland AJ et al: Deep PeronealNerve Injury as a Result of Arthroscopic Meniscectomy: A Case Report and Review of the Literature. JBJS 75A (8): 1221-1224, 1993.

10. Peicha G, Pascher A, Schwarzl F et al. Transection of the Peroneal Nerve Complicating Knee Arthroscopy: Case Report and Cadaveric Study. Arthroscopy 14: 221-223, 1998.

11. Gibson M, Barnes MR, Allen MJ et al: Weakness of foot dorsiflexion and changes in compartment pressures after tibial osteotomy. JBJS 68B (3): 471-475, 1986.

12. Kirgis A, Albrecht $\mathrm{S}$ et al: Palsy of the deep peroneal nerve after proximal tibial osteotomy: An anatomical study. JBJS 74A (8): 1180-1185, 1992.

13. Robinson CM, O’Donnell J, Will E et al: Dropped Hallux after Intramedullary Nailing of Tibial Fractures. JBJS 81B (3): 481-484, 1999.

14. Miller DB. Arthroscopic meniscus repair. Am J Sports Med 16 (4): 315-320, 1988.

15. Deutsch A, Wyzykowski RJ, Victoroff BN et al: Evaluation of the Anatomy of the Common Peroneal Nerve: Defining Nerve-at-risk in Arthroscopically Assisted Lateral Meniscus Repair. Am J of Sports Med 27 (1): 10-15, 1999.

16. Elgafy H, Ebrahaim NA, Shaheen PE et al. Extensor Hallucis Longus Innervation: An Anatomic Study. Clin Orthop 398: 245-251, May, 2002. 